\author{
Gregory W. Hall * \\ Jeff R. Crandall \\ Gregory S. Klopp \\ Walter D. Pilkey \\ Department of Mechanical, \\ Aerospace, and Nuclear \\ Engineering \\ Automobile Safety Laboratory \\ University of Virginia \\ 1011 Linden Avenue \\ Charlottesville, VA 22902, USA
}

\section{Angular Rate Sensor Joint Kinematics Applications}

High speed rotary motion of complex joints were quantified with triaxial angular rate sensors. Angular rate sensors were mounted to rigid links on either side of a joint to measure angular velocities about three orthogonal sensor axes. After collecting the data, the angular velocity vector of each sensor was transformed to local link axes and integrated to obtain the incremental change in angular position for each time step. Using the angular position time histories, a transformation matrix between the reference frame of each link was calculated. Incremental Eulerian rotations from the transformation matrix were calculated using an axis system defined for the joint. Summation of the incremental Eulerian rotations produced the angular position of the joint in terms of the standard axes. This procedure is illustrated by applying it to joint motion of the ankle, the spine, and the neck of crash dummies during impact tests. The methodology exhibited an accuracy of less than $5 \%$ error, improved flexibility over photographic techniques, and the ability to examine 3-dimensional motion.

\section{INTRODUCTION}

The selection of sensors for the analysis of joint motion during impact loading depends largely on the type of joint to be analyzed. A direct relationship between the complexity of the joint and the complexity of the instrumentation required to analyze joint motion may be established. For example, a simple hinge joint requires one rotary potentiometer mounted in line with the axis of the joint. Increased information is required for the motion analysis of a more complex joint, such

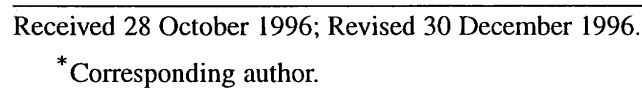

Shock and Vibration, Vol. 4, No. 4, pp. 223-229 (1997) ISSN 1070-9622/97/\$8.00 ㄷ 1997 IOS Press as the human neck, which is composed of eight, multiple degree of freedom joints.

To quantify the motion of a 6 degree of freedom joint, the three-dimensional (3-D) orientation of links on either side of the joint must be measured. Historically, 3-D motion of a joint has been observed via film analysis. Orientation information is determined by tracking the Cartesian location of three points on each rigid body with two or more views. One advantage of this method is that it does not affect the behavior of the joint. Film analysis, however, is subject to parallax, photo target placement inaccuracies, and op- 
erator errors. Film analysis during impact loading also requires high speed cameras and a minimum of two synchronous views of the 3-D joint in question.

Other orientation measurement methods operate on either sonic or magnetic field principles. Siegler et al. (1988) determined a 3-D link location by digitizing the signal from sonic emitters placed on joint links. They reported a maximum positional error of $0.4 \mathrm{~mm}$ within the working volume of their experiment. This seems like an accurate positioning method, but the actual size of the working volume and corresponding joint orientation accuracy were not stated by the authors.

Another method, commercially known as the "flock of birds" technique (Ascension Technology Corporation, Burlington, VT), capitalizes on the properties of a pulsed DC magnetic field to determine the location of receivers mounted on the links (An et al., 1987). The advantages of this system are that the magnetic field will permeate all nonmetallic objects and that the magnetic field measurements may be made by multiple receivers simultaneously. This process has a maximum sampling rate of $144 \mathrm{~Hz}$ and can be affected by metallic objects in the test area. This system is limited to a workspace with a $3-\mathrm{m}$ radius but can provide 2-mm translational accuracy and $0.5^{\circ}$ rotational accuracy.

Joint motion may also be measured by an electrogoniometer (Penny and Giles Biometrics Ltd., Gwent, UK, model M-110 Twin Axis Goniometer) mounted in parallel with the joint. These devices flex with the joint while sensing the angle of flexion with a strainsensing element. The stiffness of the flexible measurement element is extremely low to prevent interference with joint motion. Current electrogoniometers measure motion about two orthogonal axes while permitting some extension between the joint links. A second instrument would be required for 3-D studies. Durability and cross-talk sensitivity between the axes have been found to be electrogoniometer weaknesses (Hall et al., 1996).

With the development of small, light weight sensors, it has become possible to mount acceleration and angular rate sensors to rigid bodies and to measure the corresponding parameters in a local coordinate frame. Three-dimensional orientation of a rigid body may be measured using a nine accelerometer array or a triaxial angular rate sensor (Padgaonkar et al., 1975; Shipley and Baughn, 1993). The advantages of this approach are that data are presented in a local coordinate system, a view of the link is not required during testing, there is no cross-axis interference of the signal, and the sensors employed are durable. The disadvantages of this method are that the sensors and their mounts affect the mass and inertial properties of the joint links and that data must be postprocessed to obtain angles of rotation. This approach is only appropriate for dynamic joint tests.

Although each method of joint analysis has merit, the most durable and flexible system for impact testing was determined to be the method of mounting sensors to the joint links. Sonic and magnetic field techniques seem promising, but their limited respective functional range and sensitivity to metallic materials prevented them from being viable options. Photographic techniques were eliminated from consideration because multiple views of each joint link are not always available. This article describes the mathematical basis for the angular rate sensor joint analysis approach and three examples of joints that have been analyzed.

\section{MATERIALS AND METHODS}

The research presented in this article used the magnetohydrodynamic (MHD) angular rate sensor [Applied Technology Associates (ATA), model ARS-04E Dynacube $^{\mathrm{TM}}$, Albuquerque, NM] to measure the 3-D angular rate during impact testing. Details of the function, calibration techniques, and previous applications of MHD Dynacubes may be found in Shipley and Baughn (1993) and Hall et al. (1996). All impact tests were sampled at a frequency of $10 \mathrm{kHz}$ for a duration of $200 \mathrm{~ms}$. Data was filtered to channel class $180 \mathrm{spec}-$ ifications (SAE J211, 1989).

\section{JOINT ANALYSIS PROCEDURE}

The first step in joint analysis is to define a local coordinate system for links $\mathbf{A}$ and $\mathbf{B}$, located on either side of the joint (Fig. 1). Next, an Eulerian sequence of rotations to describe the joint motion must be selected. An Eulerian sequence of rotations involves three sequentially dependent rotations about coordinate axes. The first angle defines the angle between the axis system of link $\mathbf{A}$ and the prime $\left(^{\prime}\right)$ frame. The second angle defines the angle of rotation between the prime frame and the double prime (") frame. The third angle defines the rotation from the double prime frame to the axis system of link B. Many different Eulerian sequence permutations are possible as long as no two sequential rotations are about the same axis. For an arbitrary joint, the first angle in the sequence is about the primary axis of rotation of the joint. In the case of the ankle, knee, elbow, wrist, and neck, the $y$ axis (positive $=$ left) is selected as the first axis of rotation. For side impact testing of the spine, the $x$ axis (positive $=$ anterior) is selected as the first axis of rotation. 


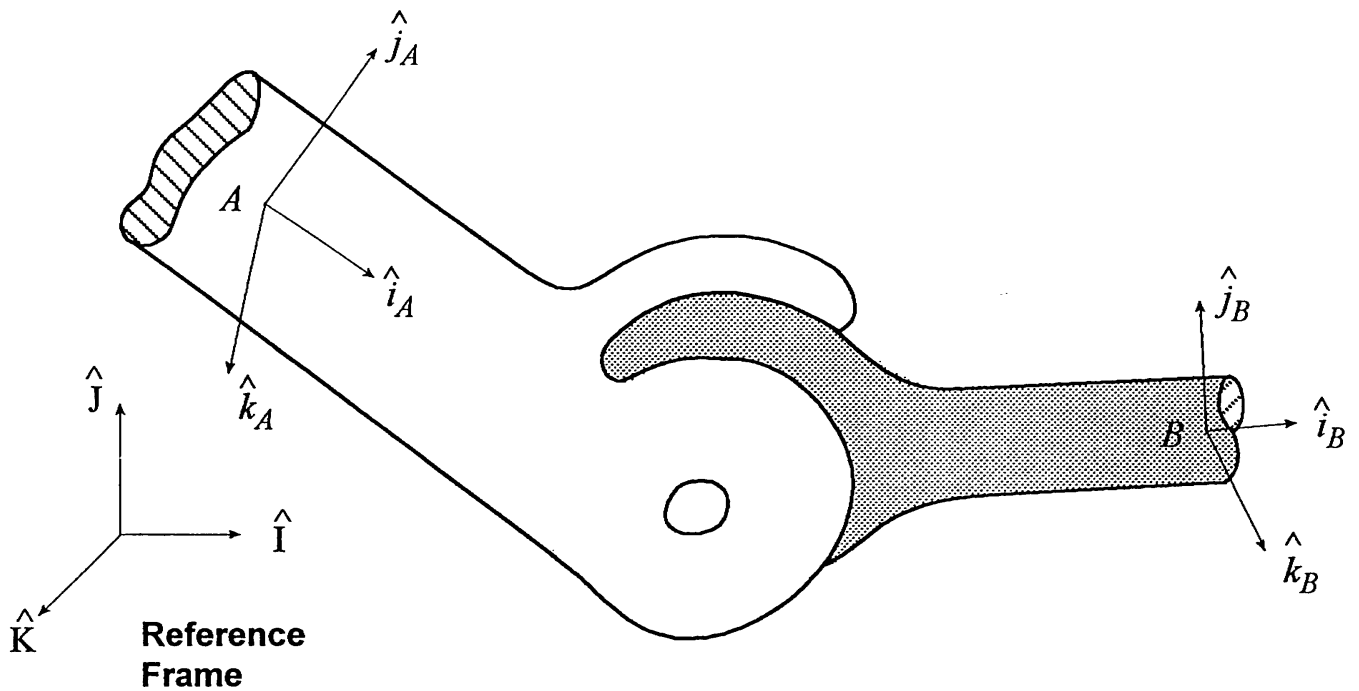

FIGURE 1 Generic joint system.

The second and third angles are selected based on the range of motion of the joint in those directions as well as the actual geometry of the joint. If, for example, it was desired to quantify the flexion of a series of three orthogonal pin joints with this technique, the sequence of the Eulerian angles would equal the sequence of the pin joints.

The use of Eulerian angles is convenient because the results are more intuitive than the values of Euler parameters. Eulerian angles are susceptible to singularity problems, however, when any angle equals $\pm \pi / 2$ radians. Thus, the zero position of all angles of the joint should be near the center of the range of motion of the joint and is defined as neutral. This minimizes the possibility of a singularity error. After selecting a neutral orientation for the joint, a sign convention for the flexion angles must be defined.

The initial orientation of each link, $\mathbf{A}$ and $\mathbf{B}$, with respect to the laboratory reference frame must be determined prior to testing. If the initial orientation of the links with respect to the laboratory reference frame can be described with one rotation about a horizontal axis, a simple inclinometer may be all that is necessary. If the initial 3-D orientation of the link is arbitrary with respect to the reference frame, it will be necessary to measure the orientation with a 3-D goniometer. In the arbitrary case, the Eulerian angle sequence from the laboratory frame to the link may by measured directly with a 3-D goniometer or indirectly by measuring the Cartesian coordinates of three points on each rigid body.

Often the initial orientation of the triaxial angular rate sensor (ARS) with respect to the link coordinate frame is orthogonal. In this case, a simple transformation matrix may be calculated that will be constant throughout the test. In the case of arbitrary placement of the triaxial ARS, an Eulerian sequence from the sensor to the laboratory frame must be identified, as was done for the joint links. Software has been written that calculates the transformation matrices from each sensor to its respective link and relates the orientation of the two links. The relationship is

$$
\left\{\begin{array}{c}
\widehat{i}_{\text {link } A} \\
\widehat{j}_{\text {link } A} \\
\widehat{k}_{\text {link } A}
\end{array}\right\}=[\mathrm{TA}]\left\{\begin{array}{l}
\widehat{I}_{\text {reference }} \\
\widehat{J}_{\text {reference }} \\
\widehat{K}_{\text {reference }}
\end{array}\right\},
$$

where TA is the transformation matrix from the global coordinate system to the local coordinate system for link A. A similar transformation matrix exists for link B. The two links are related by the equation

$$
\{\operatorname{seg} B\}=[\mathrm{TB}] \cdot[\mathrm{TA}]^{-1}\{\operatorname{seg} A\},
$$

where the inverse of [TA] is simply the transpose because it is orthogonal.

Once the link and sensor orientations are known, the test may be conducted and the data collected. A computer program was written to postprocess the angular velocity data and provide joint rotation information. Before processing the angular velocity data, the average of the pretest data is substrated from the remaining data to remove any offset. Velocity data is transformed from each sensor to the respective link and integrated using the trapezoidal rule. The integrated angular rate data (angles) are used as Eulerian angles about $x(\theta), y(\phi)$, and $z(\Psi)$ to calculate the transformation matrix of each link at time $t$ with respect to the position at the previous time $t-\Delta t$. At 
a sampling frequency of $10,000 \mathrm{~Hz}$, the magnitude of the Eulerian angles is small enough to make them independent of sequence (Hall et al., 1996; Kane et al., 1983).

Initially, the transformation matrix that relates link A to the inertial frame is set to the identity matrix, while the transformation matrix that relates link $\mathbf{B}$ to the inertial frame is set to the initial $\mathbf{A}-\mathbf{B}$ transformation matrix. The $\mathbf{A}$ frame to the inertial frame and the B frame to the inertial frame transformation matrices
[Eq. (4)] are determined for every time $t$ during the test by multiplying the respective link matrix for $t-\Delta t$ by the transformation matrix from $t-\Delta t$ to $t$. From these transformation matrices the yaw, pitch, and roll sequence of angles from the link $\mathbf{A}$ frame to the link B frame are determined for every time $t$. Joint angles are calculated about the set of orthogonal axes defined for each system. The transformation matrix for a sequence of $y-x^{\prime}-z^{\prime \prime}$ rotations relating link $\mathbf{A}$ to link B is defined as follows [Eq. (3)]:

$$
\left\{\begin{array}{c}
\widehat{i}_{B} \\
\widehat{j}_{B} \\
\widehat{k}_{B}
\end{array}\right\}=\left[\begin{array}{ccc}
\cos \phi \cos \psi+\sin \phi \sin \theta \sin \psi & \cos \theta \sin \psi & -\sin \phi \cos \psi+\cos \phi \sin \theta \sin \psi \\
-\cos \phi \sin \psi+\sin \phi \sin \theta \cos \psi & \cos \theta \cos \psi & \sin \phi \sin \psi+\cos \phi \sin \theta \cos \psi \\
\sin \phi \cos \theta & -\sin \theta & \cos \phi \cos \theta
\end{array}\right] \times\left\{\begin{array}{l}
\widehat{i}_{A} \\
\hat{j}_{A} \\
\widehat{k}_{A}
\end{array}\right\}
$$

Because the orientation of the $\mathbf{A}$ frame and $\mathbf{B}$ frame unit vectors are known at each time point from the integration and transformation of the angular rate data, the yaw ( $\phi$ about $y$ ), pitch $\left(\theta\right.$ about $\left.x^{\prime}\right)$, and roll $(\psi$ about $z^{\prime \prime}$ ) rotations for the joint in question can be calculated with

$$
\begin{aligned}
\theta & =\sin ^{-1}\left(-\vec{k}_{B} \cdot \vec{j}_{A}\right) \\
\psi & =\sin ^{-1}\left(\frac{\widehat{i}_{B} \cdot \widehat{j}_{A}}{\cos \theta}\right), \\
\phi & =\sin ^{-1}\left(\frac{\widehat{k}_{B} \cdot \widehat{j}_{A}}{\cos \theta}\right),
\end{aligned}
$$

where $-\pi / 2<\theta<\pi / 2$. The unit vector for the $\mathbf{A}$ and $\mathbf{B}$ coordinate systems are expressed in the inertial coordinate system $(\widehat{I}, \widehat{J}, \widehat{K})$ so that the dot products of the equation can be calculated. This method involves solving for three unknowns with nine equations without an optimization scheme. An optimization procedure was deemed unnecessary because of the satisfactory correlation in the experimental verification results.

\section{APPLICATION TO ANKLE JOINT}

The local coordinate system of the leg and foot were defined for a standing position, where $x$ was anterior, $z$ was superior, and $y$ was left. In the Eulerian angle sequence defined for the ankle joint (Fig. 2), flexion $(\phi)$ occurs about the $y$ axis that is fixed in the leg, $x$ version $(\theta)$ occurs about the $x$ axis that is floating (because it is the second Eulerian angle in the sequence), and axial rotation $(\psi)$ occurs about the $z$ axis of the foot. As a result of the Eulerian angle sequence, the floating $x$ axis is defined so that it moves when flexion

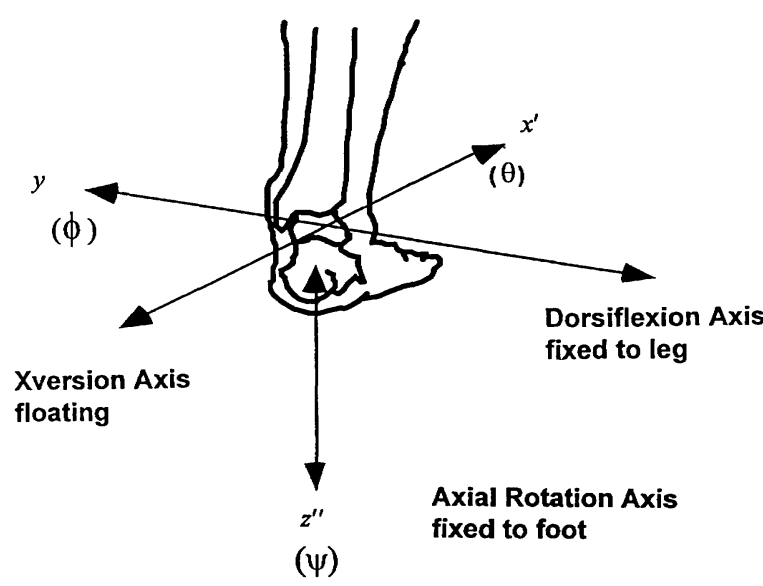

FIGURE 2 Ankle axis system.

occurs but remains stationary when axial rotation occurs. This allows the finite angular rotation of anatomical joints to be measured directly and uniquely (Chao, 1980).

Triaxial ARS cubes are mounted in an orthogonal relationship to the foot and leg of the Hybrid III dummy by modifying preexisting fasteners in the dummy lower extremity. A sign convention has been adopted for ankle motion that assigns eversion, dorsiflexion, and internal rotation as positive and inversion, plantar flexion, and external rotation as negative about the $x, y$, and $z$ axes, respectively. To comply with the sign convention, the values for internal or external rotation and eversion or inversion are negated for a right leg in the software.

The accuracy of the angular rate sensor procedure for the study of ankle joint kinematics was verified using the Advanced Lower Extremities (ALEX) for the Hybrid III crash dummy under impact loading conditions. The ALEX was chosen to verify the ARS tech- 


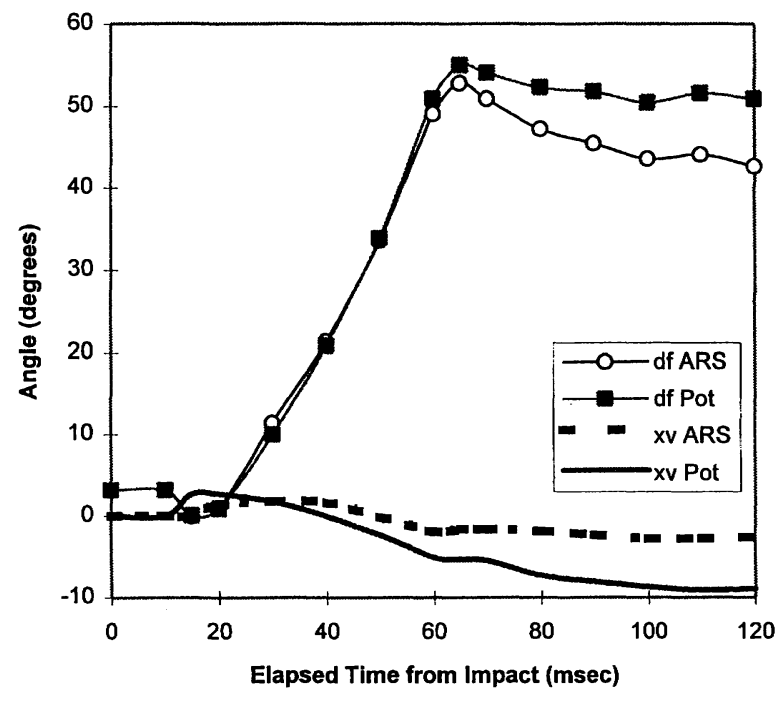

FIGURE 3 Angular rate technique verification with the ALEX.

niques because it is equipped with two orthogonal rotational potentiometers [Tokyo Cosmos Electric Company (TOCOS), model 12P2Z, Tokyo, Japan] to measure flexion and $x$ version of the ankle directly.

After mounting ARS cubes on both the foot and leg of the ALEX, impact loads were applied to the plantar surface of the foot. The results indicated a strong correlation between the ARS processed ankle data and the flexion and $x$ version potentiometers of the ALEX, with root mean square error of $\pm 2 \%$ (Fig. 3). These results seem reasonable because the sequence of pin joints in the ALEX1 is $z-y^{\prime}-x^{\prime \prime}$, instead of the convention adopted in this study.

\section{APPLICATION TO THE SPINE}

An impact test with a side impact dummy (SID) was conducted to examine the feasibility of spine flexion analysis and to study whole body rotations. Impact loads were applied to the left side of the SID with an impact pendulum (Fig. 4). The coordinate system of the spine was consistent with the human body coordinate system: $x$ is anterior, $y$ is left, and $z$ is up. Triaxial ARSs were used to track the rotary motion of the upper thoracic and lumbar spine in the laboratory frame as well as the flexion of the spine between the two sensors.

Two ARSs were mounted orthogonal to the spine of the dummy. The mounting plates were oriented in the coronal plane, with the $x$ sensor of the angular rate sensor cube pointed in the left (conventional positive $y$ ) direction of the dummy. The relationship be-

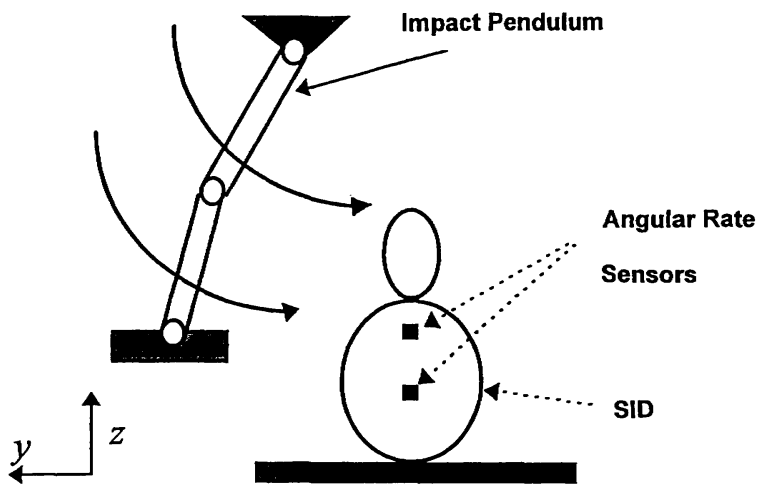

FIGURE 4 Side impact test design.

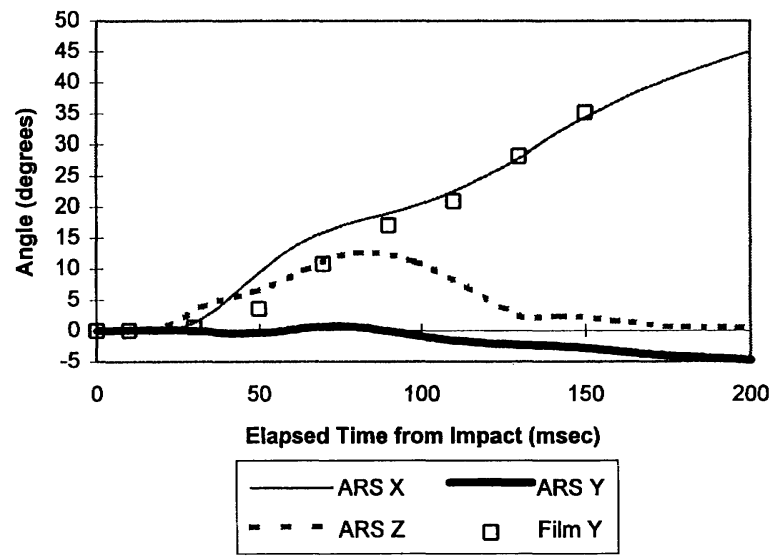

FIGURE 5 Thoracic angular rate sensor results in the laboratory frame.

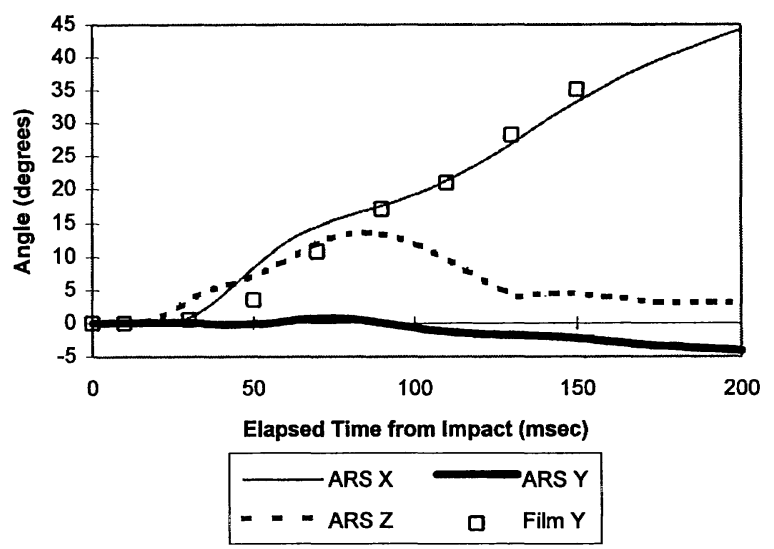

FIGURE 6 Lumbar angular rate sensor results in the laboratory frame.

tween the sensors and the spinal segments was described by

$$
\left\{\begin{array}{c}
\widehat{i}_{\text {seg }} \\
\widehat{j}_{\text {seg }} \\
\widehat{k}_{\text {seg }}
\end{array}\right\}=\left[\begin{array}{ccc}
0 & 1 & 0 \\
0 & 0 & 1 \\
-1 & 0 & 0
\end{array}\right]\left\{\begin{array}{l}
\widehat{i}_{\text {sen }} \\
\widehat{j}_{\text {sen }} \\
\widehat{k}_{\text {sen }}
\end{array}\right\} .
$$




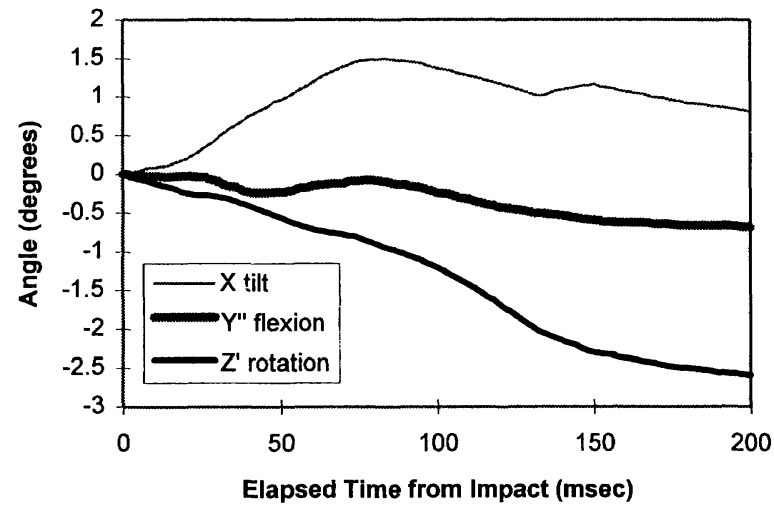

FIGURE 7 Spinal motion after pendulum impact.

The initial orientation of both the upper and lower spinal segments was determined with a 3-D goniometer, a device that measures a set of three Euler angles from the fixed laboratory frame to the local sensor frame. Postimpact angular velocities of the upper and lower torso of the dummy were recorded by the ARSs at a rate of 10,000 samples per second.

The body rotation data from the ARSs compared well with high speed films of the $x y$ plane. Film analysis was limited to 2 dimensions, while the angular rate sensors provided 3-D analysis capabilities (Figs. 5, 6).

With 3-D rotational information at two points on the spine, the response of the spine was examined. The main motion of the spine of the SID can be observed by defining an Eulerian angle sequence for the spine and using the transformation matrix from the lumbar sensor to the thoracic sensor. The angle sequence applied was a tilt about the $x$ axis, followed by rotation about the $z^{\prime}$ axis, then flexion about the $y^{\prime \prime}$ axis (Fig. 7).

\section{NECK FLEXION}

Angular rate data were collected during airbag deployments to the chest of a fifth percentile female Hybrid III dummy. The axis system for the head, as shown in Fig. 1, is defined with the $x$ axis in the anterior direction, the $y$ axis in the left direction, and the $z$ axis in the superior direction. Neutral neck orientation was consistent with the standing position. Segment A was defined as the upper thoracic spine, while segment B was defined as the head. Neck motion occurred in sequence about the $y, x^{\prime}$, and $z^{\prime \prime}$ axes from the spine to the neck, with polarities assigned in accordance with Fig. 8.

One ARS cube was mounted on the posterior head plate. A second ARS cube mount was attached to the
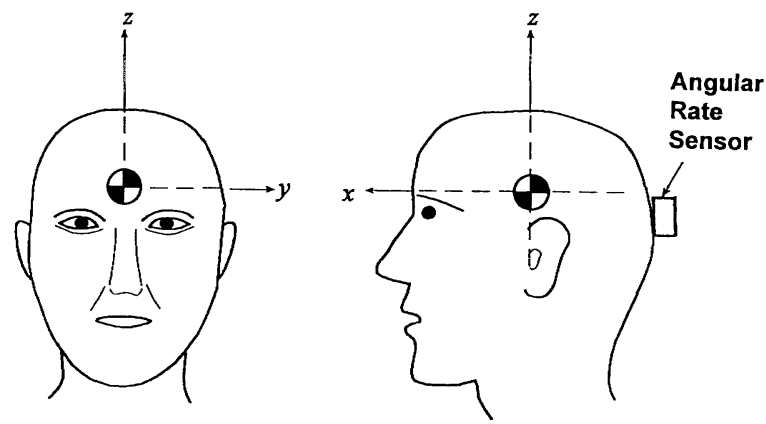

FIGURE 8 Head coordinate system.

upper spine of the dummy with preexisting fasteners. Both sensors were mounted orthogonally with respect to their segment coordinate systems so that a simple transformation matrix could be calculated for transforming sensor data into data in the local segment coordinate system. The transformation matrix for both the head and spine was

$$
\left\{\begin{array}{c}
\widehat{i}_{\text {seg }} \\
\widehat{j}_{\text {seg }} \\
\widehat{k}_{\text {seg }}
\end{array}\right\}=\left[\begin{array}{ccc}
0 & 1 & 0 \\
0 & 0 & 1 \\
-1 & 0 & 0
\end{array}\right]\left\{\begin{array}{c}
\widehat{i}_{\text {sen }} \\
\widehat{j}_{\text {sen }} \\
\widehat{k}_{\text {sen }}
\end{array}\right\} .
$$

During pretest preparation, the rotation of the head and spine about the laboratory $x$ and $z$ axes was minimized (equal to zero during postprocessing). Initial orientation of the head and thoracic sensors about the $y$ axis was measured with an inclinometer. During the test, angular rate data were sampled at a frequency of $10 \mathrm{kHz}$ and postprocessed with a specially written neck flexion computer code. The neck motion results for one of the air bag tests are shown in Fig. 9.

\section{DISCUSSION AND CONCLUSIONS}

For multilinked joints, an Eulerian sequence of rotations does not completely describe the status of the entire segment being analyzed. In neck impact tests, for example, the flexion angles indicated the relative orientation of the head to the upper thorax of the dummy. In a case of forward translation of the head, the neck joints flexed near the thorax and extended near the head, but the overall relationship between the head and thorax was reported as zero flexion. In other words, the Eulerian angle sequence describes the relationship between two link reference frames but does not necessarily describe the orientation of each joint in the multilinked application.

There are several advantages to using the ARS over film for impact research:

1. the ARS measures data in real time, 


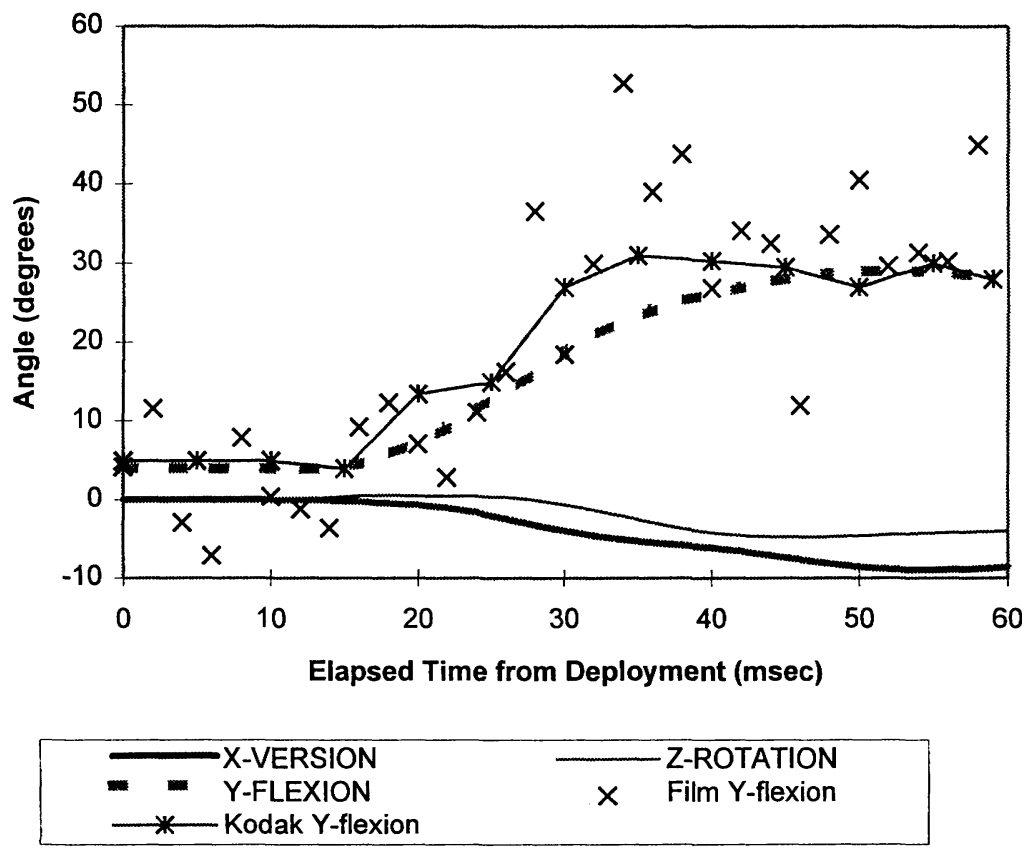

FIGURE 9 Neck motion after airbag deployment.

2. postprocessing is automated, and

3. 3-D information is obtained.

Accuracy was verified by demonstrating that the ARS results agreed to within $2 \%$ of ankle potentiometer data from an anthropomorphic crash dummy. Tests on the spine and neck demonstrated that ARS was more accurate than photographic methods because the sensor was fastened to the rigid body while photographic analysis necessitated an unobstructed view of the test.

\section{REFERENCES}

An, K. N., Jacobson, M. C., Berglund, L. J., and Chao, E. Y., 1988, "Application of a Magnetic Tracking Device to Kinesiologic Studies," Journal of Biomechanics, Vol. 21, pp. 613-620.

Chao, E. Y., 1980, "Justification of Triaxial Goniometer for the Measurement of Joint Rotation," Journal of Biomechanics, Vol. 13, pp. 989-1006.

Hall, G. W., Crandall, J. R., Klisch, S. M., Klopp, G. S., and Pilkey, W. D., 1996, "Measurement of Dynamic Joint Motion Using Magnetohydrodynamic Angular Rate Sensors," The Shock and Vibration Digest, Vol. 28, pp. 12-17.

Kane, T., Lilkins, P., and Levinson, D., 1983, Spacecraft Dynamics, McGraw-Hill, New York.

Padgaonkar, A. J., Krieger, K. W., and King, A. I., 1975, "Measurement of Angular Acceleration of a Rigid Body Using Linear Accelerometers," Journal of Applied Mechanics, Vol. 42, pp. 552-556.

SAE, 1989, "SAE Recommended Practice SAE J211 JUN88-Instrumentation for Impact Test," SAE Handbook, Vol. 4: On-Highway Vehicles and Off-Highway Machinery, Society of Automotive Engineers, New York.

Shipley, B. W., and Baughn, D. J., 1993, "An Evaluation of Sensor Systems for Measuring Rotational Motion," Proceedings of the 31st Annual SAFE Symposium, SAFE Association, Nashville, TN, pp. 127-135.

Siegler, S., Chen, J., and Schneck, C. D., 1988, "The ThreeDimensional Kinematics and Flexibility Characteristics of the Human Ankle and Subtalar Joints-Part 1: Kinematics," Journal of Biomechanical Eng., Vol. 110, pp. 364385. 

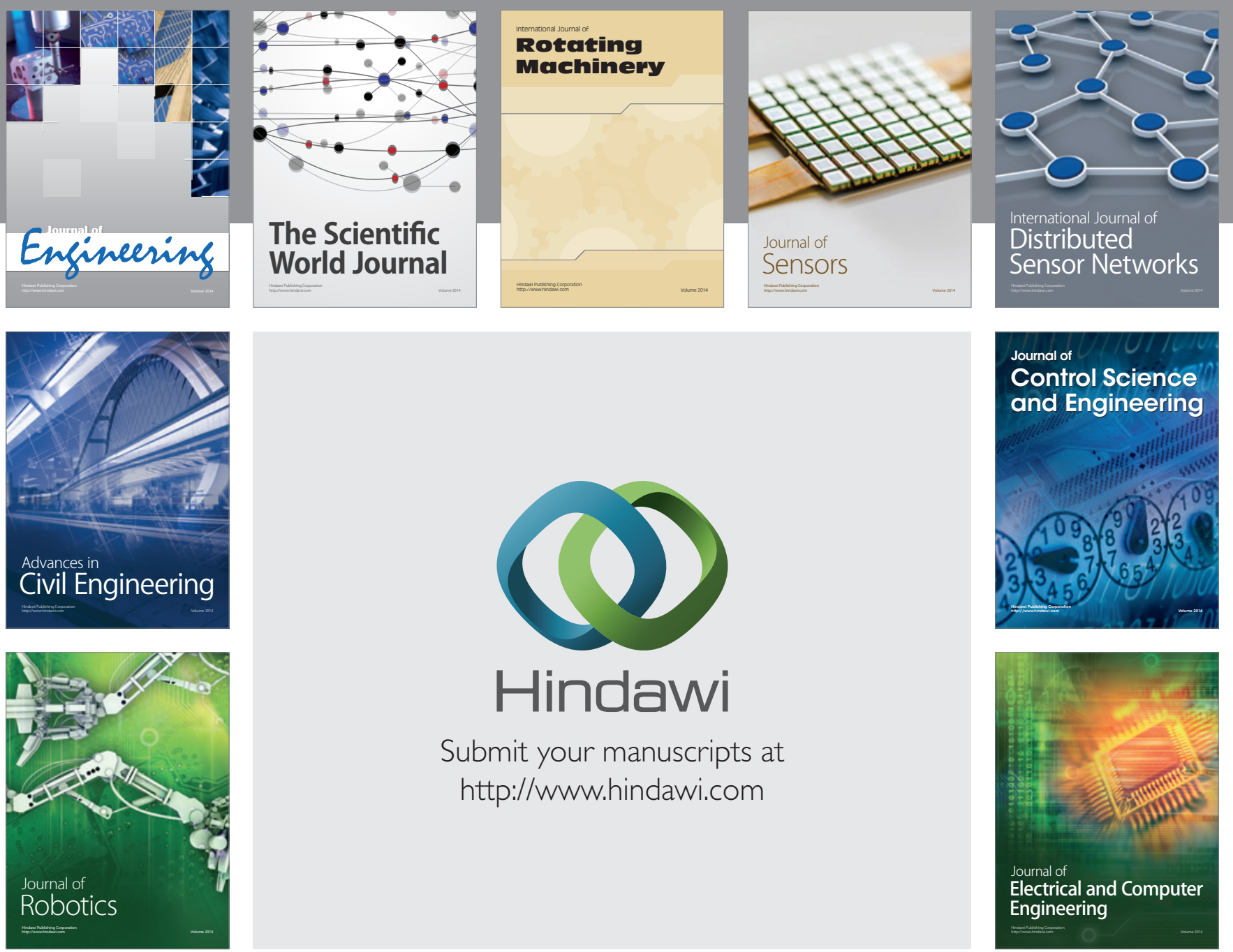

Submit your manuscripts at

http://www.hindawi.com
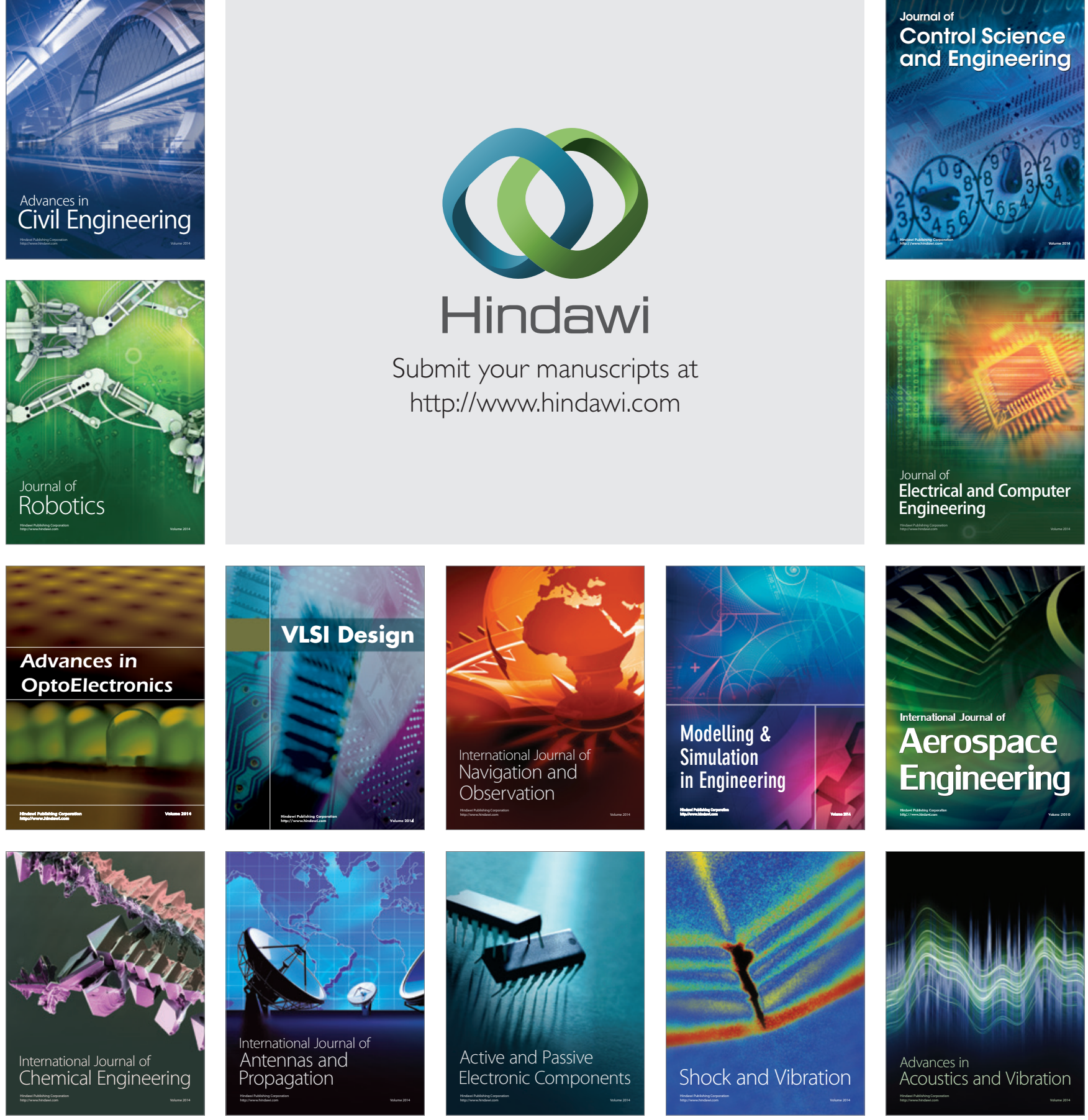